\title{
Blossoming Analysis of Composite Deployable Booms
}

\author{
Sicong Wang ${ }^{\mathrm{a}, *}$, Mark Schenk $^{\mathrm{b}}$, Shengyuan Jiang ${ }^{\mathrm{a}}$, Andrew Viquerat ${ }^{\mathrm{c}}$ \\ ${ }^{a}$ Harbin Institute of Technology, Harbin, China, 150001 \\ ${ }^{b}$ Bristol Composites Institute, University of Bristol, Bristol, England, BS8 1QU \\ ${ }^{c}$ University of Surrey, Guildford, Surrey, England, GU2 7XH
}

\begin{abstract}
Deployable coilable booms are extendible structures that have been used in a number of space and terrestrial applications. Fiber reinforced polymer (FRP) composite variants of these deployable booms have advantages over the metallic versions in the form of higher specific stiffness and greater design flexibility. A deployment failure mode called 'blossoming', in which the boom unwinds and extends within the deployment mechanism, can occur if an excessive load is applied to the boom tip. Blossoming can be mitigated by using compression rollers which radially constrain the coiled boom. An energy method is used to model the composite boom during deployment, and to predict the tip force a boom can withstand before blossoming occurs. The analytical results are compared with experimental results. The effects of the boom material properties and geometric parameters are investigated to provide more guidance in the design of deployable coilable boom systems.

Keywords: Deployable boom; blossoming; composite
\end{abstract}

\section{Introduction}

Deployable coilable booms are extendible structures used in situations in which low mass, a simple and reliable deployment mechanism, and a small storage volume are desirable. They are found in spacecraft structures such as antennas,

\footnotetext{
${ }^{*}$ Corresponding author

URL: wangsicong890101@126.com (Sicong Wang)
}

Preprint submitted to Thin-Walled Structures

September 4, 2020 


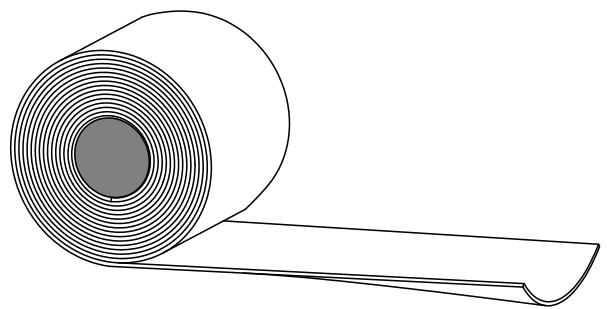

Figure 1: Deploying STEM slit boom.

solar sails and solar panels. A typical example is shown in Figure 1. A version of these booms, called the Storable Tubular Extendable Member (STEM), was first used by Klein for the antenna on the satellite Alouette [1]. Such slittube booms can be coiled on a central hub and deploy much like a carpenter's tape. A variety of coilable booms with different deployed cross-sections have been developed to match various bending and torsional stiffness requirements, including the 'lenticular' closed-section design, and the Triangular Rollable And Collapsible (TRAC) booms $[3,17]$.

Much of the early work in designing and modelling deployable coilable booms focused on isotropic materials. In recent years, booms manufactured using composite laminate materials such as carbon fiber and glass fiber have been developed due to their higher specific properties and design flexibility [4]. By choosing appropriate fiber angles within these fiber reinforced polymer (FRP) composites, some booms can be made to exhibit bistability, in which booms are not only stable in their deployed state, but also in their coiled configuration. The deployment process of mono-stable booms is likely to be highly energetic and can become chaotic. Thus, complex mechanisms are required to control the deployment, adding to the system mass and risk of deployment failure. Bistable booms can be designed in such a way that their deployment is more deterministic, and the mechanisms required to constrain the coiled booms are lighter and less complicated $[5,14,22]$. Theoretical models of composite booms have been developed to predict their natural coiled radius (also called fold radius) in the stowed state $[2,13,8,16]$. 
A typical deployment process of a coilable boom is shown in Figure 2. The boom is coiled around the central hub and the boom tip deploys outwards when the hub turns. Spring-loaded compression rollers contain the coil and prevent gaps between the coil layers as the hub rotates. The number of compression rollers will affect the force distribution between the coil layers; commonly, four rollers have been used in space sail deployment systems [15, 20]. 'Blossoming' is a failure mode that can occur during the deployment process in which the boom unwinds within the deployment mechanism by increasing its coiled radius instead of pushing the tip outwards [9]. One cause of blossoming is an excessive load on the tip. The allowable tip force is the maximum value a boom can withstand before blossoming. Predicting the tip force is essential in designing an effective deployment system. Hoskin established an energy model for isotropic booms and obtained the tip force that can be sustained during the deployment process $[10,11]$. Hoskin subsequently analyzed the relative movement of the coil layers while blossoming, and proposed a method to incorporate the effect of friction into the model; however, the work was limited to mono-stable booms [9]. Hoskin also assumed that the force distribution between the coil layers was approximately uniform, with the force applied by the compression rollers around the outside of the coil being used to derive the value of the inter-layer force. This model was later improved by incorporating a more accurate force distribution derived using finite element analysis [21].

Whereas prior work $[11,21]$ was restricted to modelling isotropic materials, in this paper we introduce the necessary components to analyze the blossoming behavior of bistable FRP booms that have flown on recent satellite missions [19]. This also allows us to compare the blossoming performance for a bistable FRP boom and an equivalent mono-stable isotropic boom. Specifically, we establish and validate an analytical model for the blossoming of coiled FRP booms that incorporates a more refined structural model (stretching components in the strain energy equations) as well as an anisotropic material model (for both symmetric and anti-symmetric laminates typically used in coilable FRP booms) [13]. Further, we demonstrate that this model can be applied to booms with a varying 


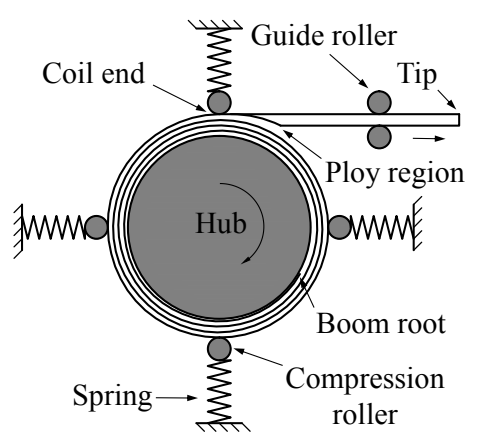

(a) nominal deployment

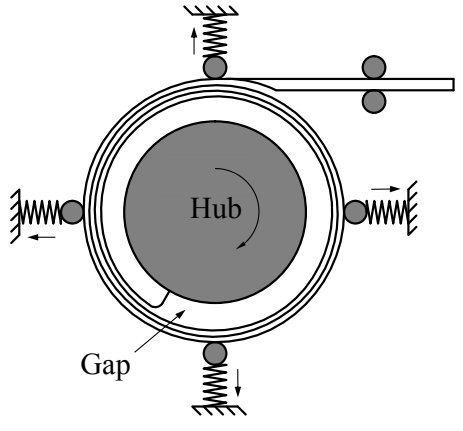

(b) blossoming

Figure 2: Deployment process of a coilable boom. Under normal deployment, the boom tip extends as the hub is rotated. Blossoming occurs when the coil expands radially, resulting in limited and unpredictable tip deployment.

ply angle. Section 2 establishes an analytical model for FRP composite booms, from which the tip force is calculated. The details of an experiment to provide practical verification are given in Section 3. In Section 4, a parametric study of the boom and the deployment mechanism is presented. Section 5 concludes the paper.

\section{Blossoming analysis of an FRP composite boom}

A diagram illustrating the boom parameters is shown in Figure 3. The centers of curvature for $\kappa_{x}$ and $\kappa_{y}$ lie on the same side of the coil, producing a synclastic (sometimes called equal-sense) curvature condition. Note that the following analysis can be adapted for opposite-sense curvatures. Using Classical Lamination Theory (CLT), the elastic behavior of the mid-surface of a composite shell can be expressed using an $A B D$ matrix as [12]:

$$
\left[\begin{array}{c}
N_{x} \\
N_{y} \\
N_{x y} \\
\hline M_{x} \\
M_{y} \\
M_{x y}
\end{array}\right]=\left[\begin{array}{ccc|ccc}
A_{11} & A_{12} & A_{16} & B_{11} & B_{12} & B_{16} \\
A_{12} & A_{22} & A_{26} & B_{12} & B_{22} & B_{26} \\
A_{16} & A_{26} & A_{66} & B_{16} & B_{26} & B_{66} \\
\hline B_{11} & B_{12} & B_{16} & D_{11} & D_{12} & D_{16} \\
B_{12} & B_{22} & B_{26} & D_{12} & D_{22} & D_{26} \\
B_{16} & B_{26} & B_{66} & D_{16} & D_{26} & D_{66}
\end{array}\right]\left[\begin{array}{c}
\epsilon_{x} \\
\epsilon_{y} \\
\gamma_{x y} \\
\hline \kappa_{x} \\
\kappa_{y} \\
\kappa_{x y}
\end{array}\right]
$$




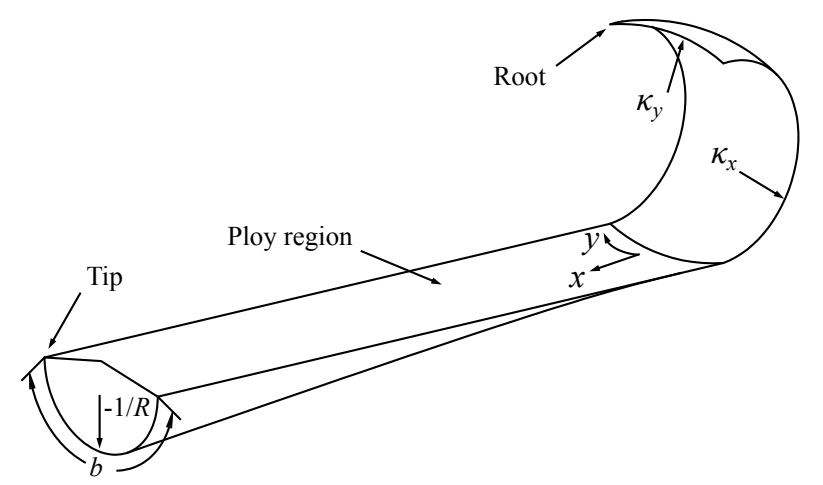

Figure 3: Boom parameters diagram

which can be expressed more compactly as:

$$
\left[\begin{array}{l}
\mathbf{N} \\
\hline \mathbf{M}
\end{array}\right]=\left[\begin{array}{l|l}
\mathbf{A} & \mathbf{B} \\
\hline \mathbf{B} & \mathbf{D}
\end{array}\right]\left[\begin{array}{c}
\epsilon \\
\hline \kappa
\end{array}\right]
$$

in which $N_{x}, N_{y}, N_{x y}$ and $M_{x}, M_{y}, M_{x y}$ are the in-plane loads and bending moments per unit length along the shell edge; $\epsilon_{x}, \epsilon_{y}$ and $\gamma_{x y}$ are the direct and shear strain, and $\kappa_{x}, \kappa_{y}, \kappa_{x y}$ are the bending and twisting curvatures.

When the boom laminate layup is symmetric, the $\mathbf{B}$ matrix is zero, which means bending and stretching are decoupled. Although the $D_{16}$ and $D_{26}$ are typically not zero, and hence bending and twisting are coupled, the coupling is resisted by the deployment mechanism. When the laminate is anti-symmetric, $D_{16}=D_{26}=0$ while $\mathbf{B} \neq 0$. However, the coupling between bending and stretching (B) has a weak effect on the deployment of the boom [13]. Since twisting has a small effect on bending behavior in both symmetric and antisymmetric cases, $\kappa_{x y}$ (and hence $D_{66}$ ) is ignored. For the particular laminate under consideration in this work, woven braided plies top and bottom ensure that $\mathbf{B}=0, D_{16}=D_{26}=0$, and $D_{66}$ can be ignored. Therefore, bending behavior in Equation 2 can be reduced to:

$$
\left[\begin{array}{l}
M_{x} \\
M_{y}
\end{array}\right]=\left[\begin{array}{ll}
D_{11} & D_{12} \\
D_{12} & D_{22}
\end{array}\right]\left[\begin{array}{l}
\kappa_{x} \\
\kappa_{y}
\end{array}\right]
$$


It may further be assumed that significant strains only occurs along the longitudinal $(x)$ axis of the boom, resulting in:

$$
N_{x}=A_{11} \epsilon_{x}
$$

According to the simplification in Equations 3 and 4, the bending and stretching strain energy per unit length of a composite shell is given as [18]:

$$
\begin{aligned}
U_{B} & =\frac{b}{2}\left[\begin{array}{lll}
\kappa_{x} & \kappa_{y} & \kappa_{x y}
\end{array}\right] \mathbf{D}\left[\begin{array}{c}
\kappa_{x} \\
\kappa_{y} \\
\kappa_{x y}
\end{array}\right] \\
& =\frac{b}{2}\left(D_{11} \Delta \kappa_{x}^{2}+2 D_{12} \Delta \kappa_{x} \Delta \kappa_{y}+D_{22} \Delta \kappa_{y}^{2}\right)
\end{aligned}
$$

and

$$
U_{S}=\frac{b}{2}\left[\begin{array}{lll}
\epsilon_{x} & \epsilon_{y} & \gamma_{x y}
\end{array}\right] \mathbf{A}\left[\begin{array}{c}
\epsilon_{x} \\
\epsilon_{y} \\
\gamma_{x y}
\end{array}\right]=\frac{b}{2} A_{11} \epsilon_{x}^{2}
$$

in which $b$ is the arc length along the cross-section. The boom strain energy per unit length $e$ can be expressed as [13]:

$$
\begin{aligned}
e= & \frac{b}{2}\left(D_{11} \kappa_{x}^{2}+2 D_{12} \kappa_{x}\left(\kappa_{y}-\frac{1}{R}\right)+D_{22}\left(\kappa_{y}-\frac{1}{R}\right)^{2}\right) \\
& +\frac{1}{\kappa_{y}} \frac{A_{11}}{2}\left(\frac{b}{2} \frac{\kappa_{x}^{2}}{\kappa_{y}}+\frac{\sin \left(b \kappa_{y}\right)}{2} \frac{\kappa_{x}^{2}}{\kappa_{y}^{2}}-\frac{4 \sin ^{2}\left(b \kappa_{y} / 2\right)}{b} \frac{\kappa_{x}^{2}}{\kappa_{y}^{3}}\right)
\end{aligned}
$$

where $R$ is the boom initial transverse radius. The first term in Equation 7 is the expression of the boom bending energy, and the second one is the stretching energy term.

In Equation 7, $\kappa_{x}$ is taken as an independent variable whose value is determined by the hub radius around which the boom is coiled. The resulting value of $\kappa_{y}$ can be found using

$$
\frac{d e}{d \kappa_{y}}=0, \quad \frac{d^{2} e}{d \kappa_{y}^{2}}>0
$$


with a corresponding minimum energy $e_{\min }$ for a given $\kappa_{x}$. The coil geometry is assumed to be an Archimedes' spiral. The longitudinal curvature, $\kappa_{x}$, will vary slightly depending on its position within this coil, and can be expressed as a function of the winding angle $\theta$ :

$$
\kappa_{x}=\frac{1}{r_{i}+a \theta}, \quad a=\frac{t}{2 \pi}
$$

where $t$ is the coil layer thickness (spiral pitch), and $r_{i}$ is the coiling radius of the boom root.

By integrating the energy per unit length along the spiral, using the arc length formula in polar coordinates, the total strain energy of the coil $E_{b}$ can be found as:

$$
E_{b}=\int_{0}^{\theta_{w}-\theta_{h}}\left(r_{i}+a \theta\right) e_{\min }(\theta) d \theta
$$

where $\theta_{w}$ is the initial total wrap coil angle, and $\theta_{h}$ is the angle through which the hub has been rotated, measured from the boom root's initial position (Figure 4). In Equation 10, the energy of the transition zone in Figure 3 (also called ploy region) is ignored in the model. Our analysis implicitly assumes that the boom has partly deployed, which means that the length of the ploy region would remain constant throughout deployment and blossoming. The energy contained within this section can therefore be regarded as constant [11]. Incorporating further details of the ploy length is beyond the scope of the current work [16].

\subsection{Normal Deployment}

In a normal deployment process, the boom deploys by extending the tip outwards with a corresponding hub angular velocity while maintaining a compact coil with no gaps between the layers, as in Figure 4(b). In this case, $\left(\theta_{w}-\theta_{h}\right)$ decreases, while $r_{i}$ remains constant (written as $r_{i n}$ in this case). Based on the Newton-Leibniz formula and Equation 10, the boom deploying torque is:

$$
T_{\text {coil }}=\frac{d E_{b}}{d\left(\theta_{w}-\theta_{h}\right)}=\left(r_{i n}+a\left(\theta_{w}-\theta_{h}\right)\right) e_{\min }\left(\theta_{w}-\theta_{h}\right)
$$




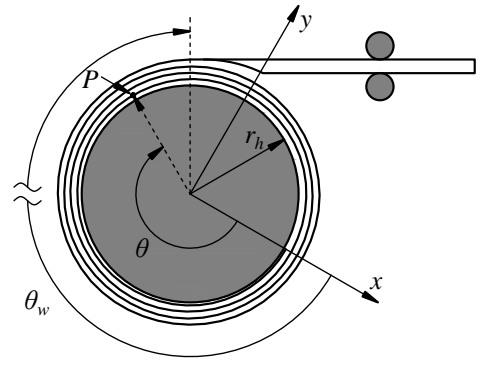

(a) initial coil geometry

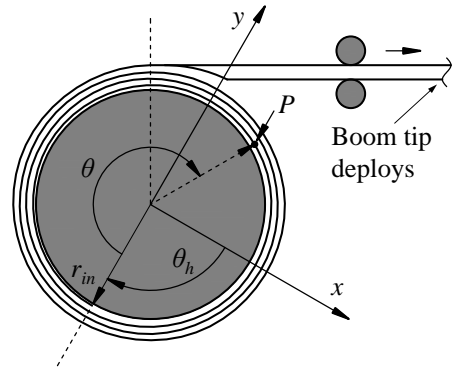

(b) normal deployment

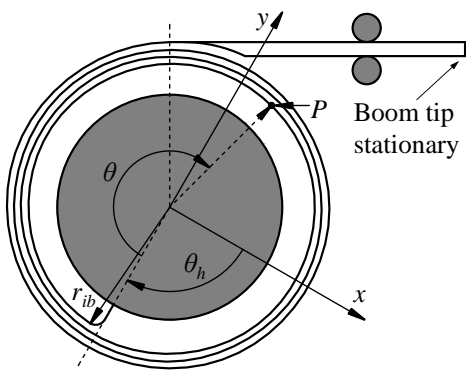

(c) blossoming

Figure 4: Coil and hub geometry definitions. Compression rollers and springs are omitted for clarity. The initial location of the boom root as it connects to the hub is used to define the $x$-axis of a non-rotating absolute coordinate system. The angle $\theta_{w}$ is the initial wrapped coil angle ( $=2 \pi n_{t 0}$ where $n_{t 0}$ is the number of turns in the initial coil) measured from the boom root to the ploy region. The angle $\theta_{h}$ is the hub rotation, measured from the $x$-axis of the absolute reference frame to the boom root. The angle $\theta$ is taken from the boom root to some point $P$ within the coil, and as such, is in the rotating reference frame of the hub. 
where $r_{i n}=r_{h}+t / 2\left(r_{h}\right.$ describes the hub radius $)$. In addition to the coil unwinding, strain energy will also be released from the springs that load the compression rollers; however, this energy is neglected during normal deployment since the change in the rollers' displacement during deployment is quite small (one boom thickness per turn).

\subsection{Blossoming}

Under normal deployment, the tip deployment is controlled by rotating the coil hub. Should the tip deployment be prevented, a continued hub rotation will cause the coil to expand radially as the total coiled length $\left(l_{\text {coil }}\right)$ remains unchanged; this process is referred to as blossoming - see Figure 4(c). In this case, a gap appears between the innermost layer and the hub, while the adjacent layers continue to rotate and slide past each other [9]. Therefore, when blossoming happens, the innermost layer radius $r_{i}$ becomes a function of the hub rotation angle $\theta_{h}$, written as $r_{i b}\left(\theta_{h}\right)$ in this case. In this process, the energy in the compression springs increases and the friction between the coil layers dissipates energy due to the relative motion of the coils. Therefore, the torque to restrain the boom from blossoming is:

$$
\begin{aligned}
T_{\text {root }} & =\frac{d\left(E_{b}+E_{s}+W_{f}\right)}{d \theta_{h}} \\
& =T_{b}+T_{s}+T_{f}
\end{aligned}
$$

where $E_{s}$ is the energy release in the compression springs (negative), $W_{f}$ is the friction work done in the blossoming process (negative); $T_{b}, T_{s}$ and $T_{f}$ are torques caused by the boom, the springs and the friction respectively.

The coiled length $l_{\text {coil }}$ is invariant during blossoming, which can be acquired from the initial state in Figure 4(a). Making use of polar coordinates, the coiled length can be obtained using the initial coil geometry as:

$$
l_{\text {coil }}=\int_{0}^{\theta_{w}} \sqrt{\left(r_{i n}+a \theta\right)^{2}+\left(\frac{d\left(r_{i n}+a \theta\right)}{d \theta}\right)^{2}} d \theta
$$


Then the dependent variable $r_{i b}\left(\theta_{h}\right)$ can be found using:

$$
l_{\text {coil }}=\int_{0}^{\theta_{w}-\theta_{h}} \sqrt{\left(r_{i b}\left(\theta_{h}\right)+a \theta\right)^{2}+a^{2}} d \theta
$$

Combining Equations 10, 12 and 14, the boom blossoming torque $T_{b}$ becomes:

$$
\begin{aligned}
T_{b} & =\frac{d E_{b}}{d \theta_{h}} \\
& =\frac{d}{d \theta_{h}} \int_{0}^{\theta_{w}-\theta_{h}}\left(r_{i b}\left(\theta_{h}\right)+a \theta\right) e_{\min }\left(\theta, \theta_{h}\right) d \theta
\end{aligned}
$$

Assuming the compression springs are placed radially, the corresponding elastic energy during blossoming is given as:

$$
\begin{aligned}
E_{s} & =n_{r}\left(\frac{1}{2} k r^{2}-\frac{1}{2} k\left(r_{\text {pre }}+\Delta r\right)^{2}\right) \\
& =-n_{r}\left(F_{\text {pre }} \Delta r+\frac{1}{2} k \Delta r^{2}\right)
\end{aligned}
$$

where $n_{r}$ is the number of compression rollers, $k$ is the spring stiffness, $r_{\text {pre }}$ is the pre-deformation of each spring, $F_{\text {pre }}$ is the pre-load on each roller and $\Delta r$ is the displacement of each roller (which is equal to the radial displacement of the outermost layer) during the blossoming process, which can be expressed as:

$$
\Delta r\left(\theta_{h}\right)=\left(r_{i b}\left(\theta_{h}\right)+a\left(\theta_{w}-\theta_{h}\right)\right)-\left(r_{i n}+a \theta_{w}\right)
$$

Combining Equations 16 and 17 into Equation 12, the torque $T_{s}$ due to the springs is found as:

$$
T_{s}=\frac{d E_{s}}{d \theta_{h}}=-n_{r} F_{r} \frac{d \Delta r\left(\theta_{h}\right)}{d \theta_{h}}
$$

where the force on each roller $F_{r}$ is a function of $\theta_{h}$ :

$$
F_{r}=F_{\text {pre }}+k \Delta r\left(\theta_{h}\right)
$$


and

$$
\frac{d \Delta r\left(\theta_{h}\right)}{d \theta_{h}}=\frac{d r_{i b}\left(\theta_{h}\right)}{d \theta_{h}}-a
$$

In order to calculate the amount of energy dissipated by friction during the blossoming process, the contact length (and hence contact area) between adjacent layers along the spiral $\left(l_{\text {con }}\right)$ can be found using:

$$
l_{\text {con }}\left(\theta_{h}\right)=\int_{0}^{\theta_{w}-\theta_{h}-2 \pi} \sqrt{\left(r_{i b}\left(\theta_{h}\right)+a \theta+\frac{t}{2}\right)^{2}+a^{2}} d \theta
$$

which is the spiral length along the coil outside surface from the innermost layer to the outermost but one layer. Finally, the effective friction torque $T_{f}$ in Equation 12 is:

$$
\begin{aligned}
T_{f}=\frac{d W_{f}}{d \theta_{h}} & =\frac{d W_{f}}{d l_{\mathrm{con}}\left(\theta_{h}\right)} \frac{d l_{\mathrm{con}}\left(\theta_{h}\right)}{d \theta_{h}} \\
& =N\left(F_{r}\right) \mu \frac{d l_{\mathrm{con}}\left(\theta_{h}\right)}{d \theta_{h}}
\end{aligned}
$$

where $\mu$ is the friction coefficient between coil layers and $N\left(F_{r}\right)$ is the integral of the pressure between layers along the coil, as function of roller force $F_{r}$.

If a deployment mechanism similar to that described in [9] is being used, the paper's approach to modelling the force acting between adjacent coil layers may also be adopted. Specifically, it is assumed that the total force acting between adjacent coil layers throughout the coil, $N\left(F_{r}\right)$, is equal to the sum of the forces applied by the compression rollers to the outermost layer, multiplied by the number of internal layers. Hence, the boom total force can be expressed as:

$$
N\left(F_{r}\right)=F_{r} n_{r}\left(n_{t}-1\right)
$$

where $n_{t}=\left(\theta_{w}-\theta_{h}\right) / 2 \pi$ is the number of coil layers/turns.

The friction torque $T_{f}$ is found by substituting Equations 21, 23 and 19 into Equation 22. Similarly, by combining Equations 15, 18 and 22 into Equation 12, the torque to restrain the boom from blossoming $T_{\text {root }}$ can be found. Following Ref. [9], if a compressive tip load is applied, the maximum load $L_{t}$ (and hence 
the tip force) can be expressed as:

$$
L_{t}\left(r_{i b}\left(\theta_{h}\right)+a\left(\theta_{w}-\theta_{h}\right)\right)=T_{\text {coil }}-\left(-T_{\text {root }}\right)
$$

In Equation 24, for a fixed coil end condition, $T_{\text {coil }}$ is rotating the hub counterclockwise, whereas $T_{\text {root }}$ is rotating the hub clockwise, which means $T_{\text {coil }}$ will be of negative sign.

Combining Equations 11, 12 and 24, the tip force value can be expressed as:

$$
F_{t}=L_{t}=\frac{T_{\text {coil }}+T_{\text {root }}}{r_{i b}\left(\theta_{h}\right)+a\left(\theta_{w}-\theta_{h}\right)}
$$

In this analysis it is assumed that the boom tip is stationary during deployment. In deployment experiments where the hub is actively rotated under an external tip load (e.g. sail deployment forces), it has been observed that the boom may simultaneously deploy and blossom. This leads to an unpredictable tip deployment, and a further rationale for mitigation of this failure mode.

\section{Experiments}

A blossoming experiment was performed to verify the analytical model. The experimental set-up is shown in Figure 5, and was identical to that used in Ref. [9]. The boom was coiled in an equal-sense direction, with the boom root fully fixed to the hub. The compression rollers applied a normal force on the outermost coil layer, while the guide rollers prevented the tip from deviating. The equivalent radial spring stiffness of the compression rollers was $k=20 \mathrm{~N} / \mathrm{m}$. After fixing the boom tip in place, blossoming was initiated by turning the hub clockwise in increments of $36^{\circ}$. A force sensor measured the tip force during blossoming.

The FRP boom was manufactured from carbon fiber and epoxy resin, and the parameters used in the following analysis were those used in the InflateSail CubeSat [20]. Three plies were used in the construction of the boom: the upper and the lower plies with braided fabric, and the unidirectional (UD) ply in the 


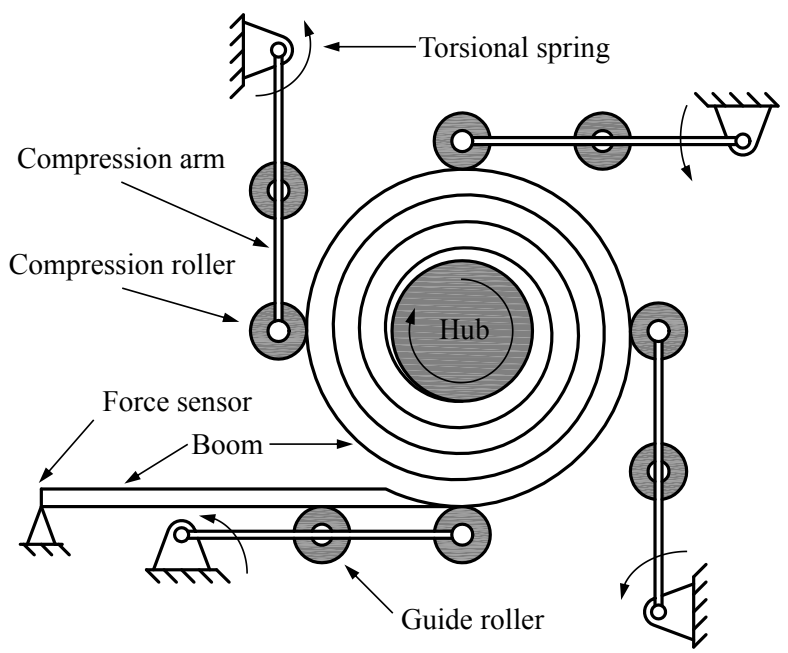

(a)

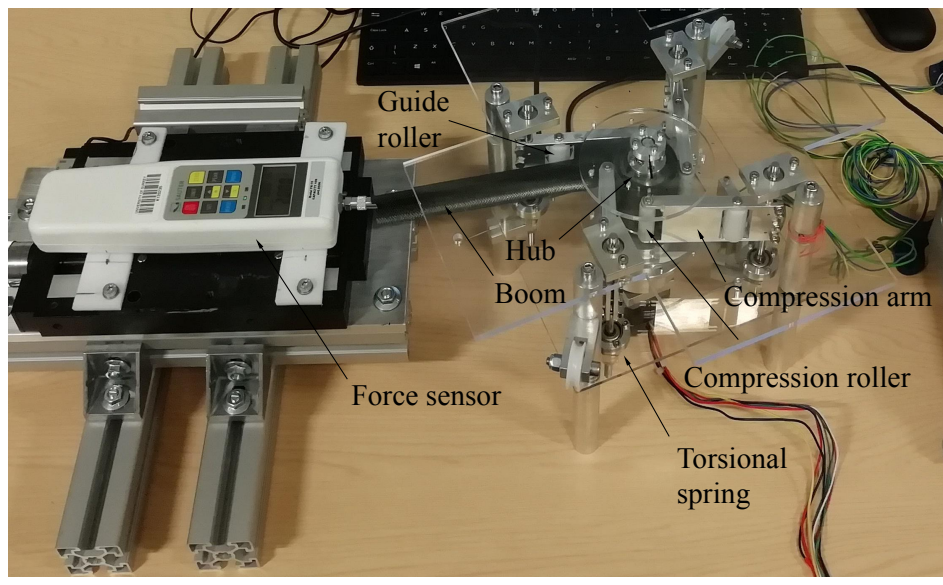

(b)

Figure 5: Experimental set-up 
middle with $0^{\circ}$ spread-tow fiber. The outer plies give the boom its bistable properties, and the UD ply provides additional longitudinal stiffness. See Ref. [6] for more details of the manufacturing process for these booms. The geometric parameters are shown in Figure 3, while the boom and the deployment mechanism values are given in Table 1. The laminate constituent material values are shown in Table 2 (in which $E_{m}, G_{m}, \nu_{m}$ are the elastic modulus, shear modulus, Poisson's ratio of the matrix, $E_{f}, G_{f}, \nu_{f}$ are the elastic modulus, shear modulus, Poisson's ratio of the fiber, $t_{\mathrm{UD}}, V_{\mathrm{UD}}, \phi_{\mathrm{UD}}$ are the thickness, volume fraction, porosity of the UD ply, and $t_{f}, V_{f}, \phi_{f}$ are the thickness (averaged), volume fraction (averaged), and porosity of each fabric ply). Ply stiffness properties were calculated using standard rules of mixtures. For the woven fabric plies, the two fiber directions are homogenised as two separate laminae with the same distance from the boom mid-surface.

Table 1: Boom and deployment mechanism parameters used in experiments

\begin{tabular}{c|c|c|c|c|c|c}
$R(\mathrm{~mm})$ & $b(\mathrm{~mm})$ & $r_{h}(\mathrm{~mm})$ & $n_{t}$ & $n_{r}$ & $F_{\text {pre }}(\mathrm{N})$ & $k(\mathrm{~N} / \mathrm{m})$ \\
\hline 20 & 50 & 20 & $15,10,5$ & 4 & $1,2,3,4$ & 20
\end{tabular}

Table 2: Laminate properties used in experiments

\begin{tabular}{c|c|c|c|c|c}
$E_{m}(\mathrm{GPa})$ & $G_{m}(\mathrm{GPa})$ & $\nu_{m}$ & $E_{f}(\mathrm{GPa})$ & $G_{f}(\mathrm{GPa})$ & $\nu_{f}$ \\
\hline 4 & 2.7 & 0.35 & 240 & 95 & 0.22 \\
$t_{\mathrm{UD}}(\mathrm{mm})$ & $V_{\mathrm{UD}}(\%)$ & $\phi_{\mathrm{UD}}(\%)$ & $t_{f}(\mathrm{~mm})$ & $V_{f}(\%)$ & $\phi_{f}(\%)$ \\
\hline 0.057 & 31 & 15 & 0.096 & 53 & 15
\end{tabular}

In this work, a Bistable Over the Whole Length (BOWL) boom with a continuously varying braided angle was used to avoid the 'fifty pence' effect for large numbers of turns (see [7]). The laminate layup varied linearly along the $3.25 \mathrm{~m}$ length of the boom, from $\left[ \pm 50^{\circ} \mathrm{F} / 0^{\circ} / \pm 50^{\circ} \mathrm{F}\right]$ at the root to $\left[ \pm 35^{\circ} \mathrm{F} / 0^{\circ} / \pm 35^{\circ} \mathrm{F}\right]$ at the tip. For the analytical calculation, the average of the braided angles at both ends of the coiled section was used. According to the analytical method 
in Section 2, the natural coiled radii of the innermost and outermost layers are $21.5 \mathrm{~mm}$ and $48.8 \mathrm{~mm}$ respectively. This means that the boom cannot be perfectly tightly coiled without some tension applied to the tip and the innermost radius is a little larger than the hub radius (20 $\mathrm{mm}$ in Table 1). Hence, a slight tension was introduced on the boom tip until the gaps between the coil layers had disappeared. For a practical design, it is required that the hub radius should be larger than the natural coiled radius to make the boom withstand a high tip force [9]. However, for the purpose of verifying the analytical model in this paper, the parameters used in the experiment are sufficient.

At the beginning of the experiment, the friction coefficient of the boom material was measured as $\mu=0.10$. For each combination of pre-load value and number of coil turns used in the experiment, three repeat tests were carried out to ensure the results were consistent. Photos of the blossoming process are shown in Figure 6, and the comparisons of the analytical and experimental results can be seen in Figures 7 to 9 .

In general, the results from the theory and the experiment matched well, with the theoretical model generally under-predicting the measured tip load. The tension applied to the boom tip to ensure tight coiling of the boom is thought to increase the friction between adjacent layers, and thus increase the tip force. Table 3 shows the mean difference between the experimental results and the theoretical predictions across the angle range of the hub rotation, for each measured combination of pre-load and number of turns. It can be seen that the analytical prediction was more accurate when the pre-load was higher. The cause of the high variation for $F_{\text {pre }}=2 \mathrm{~N}$ is currently not understood. Blossoming was less obvious when the number of turns was large - comparing Figures 6(a) and 6(b). The experimental results were less repeatable when the pre-load was low and/or the number of turns was small, because the gaps appeared not only between the innermost layer and the hub but also between the outer layers, see Figure 6(b), invalidating the assumption in the theoretical model in Section 2. On the basis of Figures 7 to 9 , it can be seen that the postblossoming tip force rose with the hub rotation. As the load on the tip should 

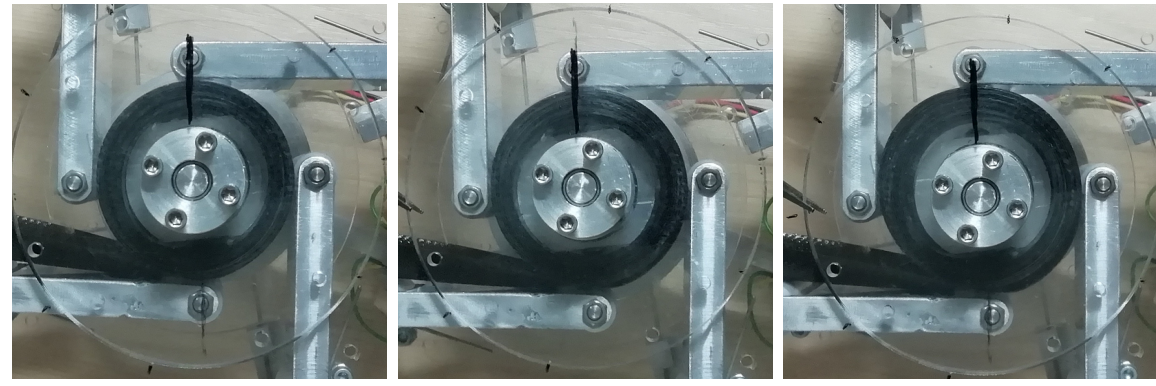

(a) blossoming of 15 turn coil with $F_{\text {pre }}=4 \mathrm{~N}$, at $0^{\circ}, 180^{\circ}$ and $360^{\circ}$
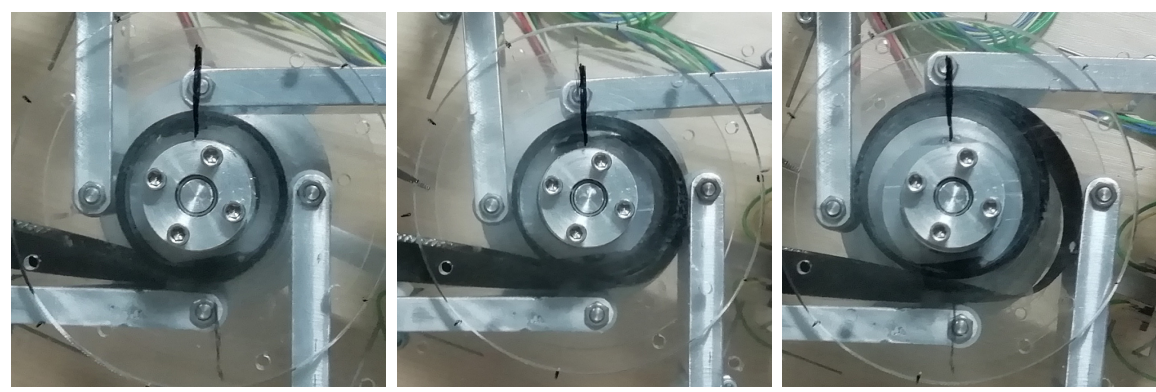

(b) blossoming of a 5 turn coil with $F_{\text {pre }}=1 \mathrm{~N}$, at $0^{\circ}, 180^{\circ}$ and $360^{\circ}$

Figure 6: Blossoming process in experiment

be lower than the lowest allowable load throughout the blossoming process in a practical design, the tip force value at the onset of blossoming should be taken as the allowable load. Meanwhile, the tip force was higher when the number of coil turns was less. Therefore, the allowable load of a boom throughout the entire deployment process should be the boom's tip force in its initial configuration. As the impact of the roller force is low, in the following analysis $F_{\text {pre }}=4 \mathrm{~N}$ was selected for the sake of illustration.

Table 3: Mean difference between experimental results and analytical predictions

\begin{tabular}{c|c|c|c|c} 
& $F_{\text {pre }}=1 \mathrm{~N}$ & $F_{\text {pre }}=2 \mathrm{~N}$ & $F_{\text {pre }}=3 \mathrm{~N}$ & $F_{\text {pre }}=4 \mathrm{~N}$ \\
\hline$n_{t}=15$ & $-0.38(-28 \%)$ & $-0.38(-22 \%)$ & $-0.36(-17 \%)$ & $-0.18(-8 \%)$ \\
\hline$n_{t}=10$ & $-0.75(-32 \%)$ & $-1.23(-38 \%)$ & $-0.67(-22 \%)$ & $-0.38(-12 \%)$ \\
\hline$n_{t}=5$ & $-0.61(-22 \%)$ & $-1.57(-37 \%)$ & $-0.39(-11 \%)$ & $-0.09(-2 \%)$
\end{tabular}




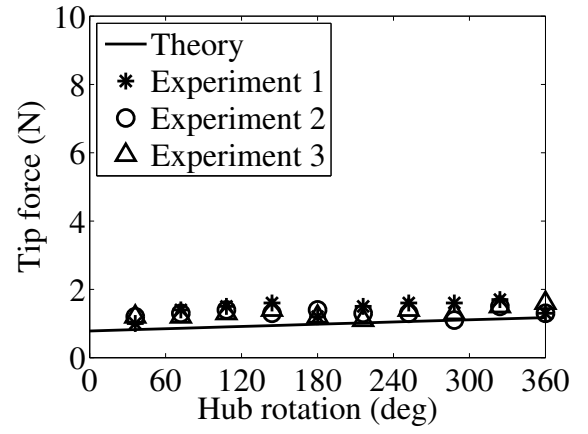

(a) $F_{\text {pre }}=1 \mathrm{~N}$

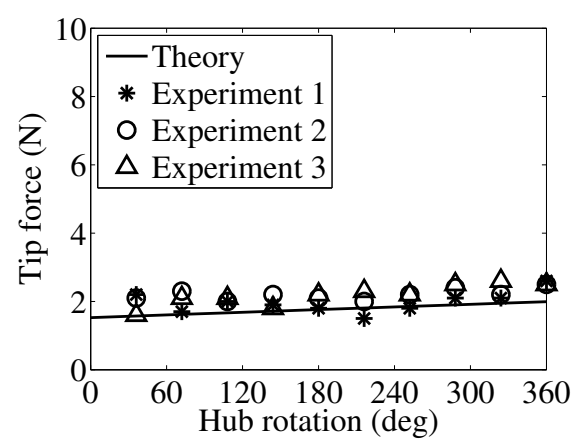

(c) $F_{\text {pre }}=3 \mathrm{~N}$

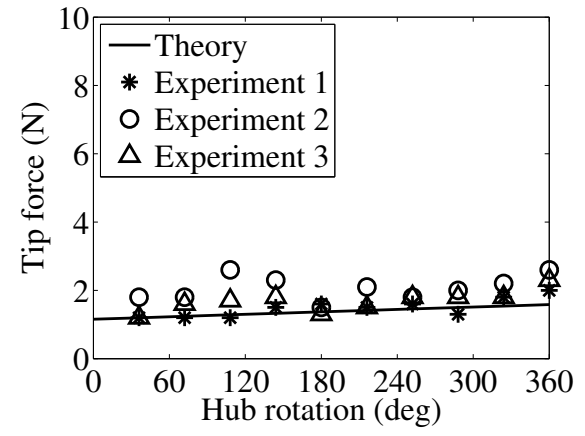

(b) $F_{\text {pre }}=2 \mathrm{~N}$

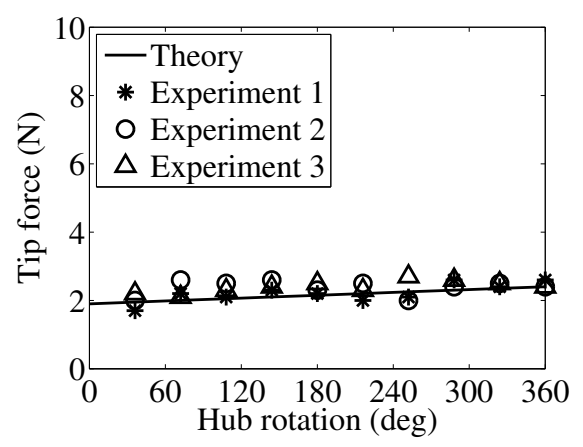

(d) $F_{\text {pre }}=4 \mathrm{~N}$

Figure 7: Tip force comparison of a 15 turns coil during blossoming $\left(\left[ \pm 45^{\circ} \mathrm{F} / 0^{\circ} / \pm 45^{\circ} \mathrm{F}\right]\right.$ (averaged), $R=20 \mathrm{~mm}, k=20 \mathrm{~N}, r_{h}=20 \mathrm{~mm}$ ) 


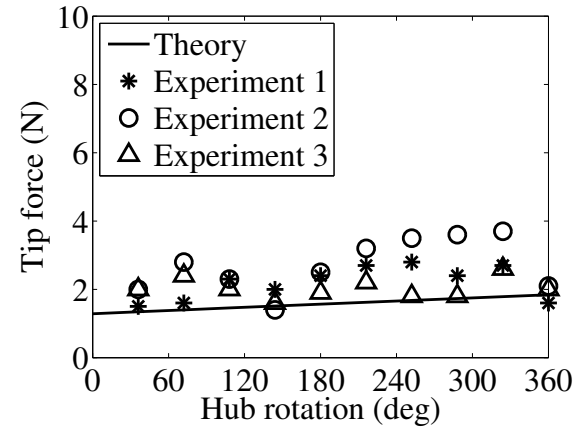

(a) $F_{\text {pre }}=1 \mathrm{~N}$

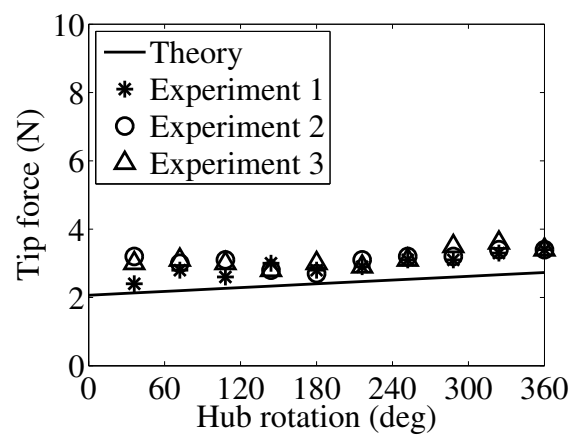

(c) $F_{\text {pre }}=3 \mathrm{~N}$

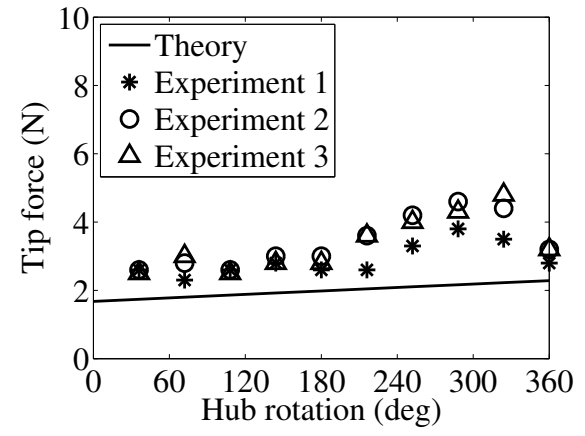

(b) $F_{\text {pre }}=2 \mathrm{~N}$

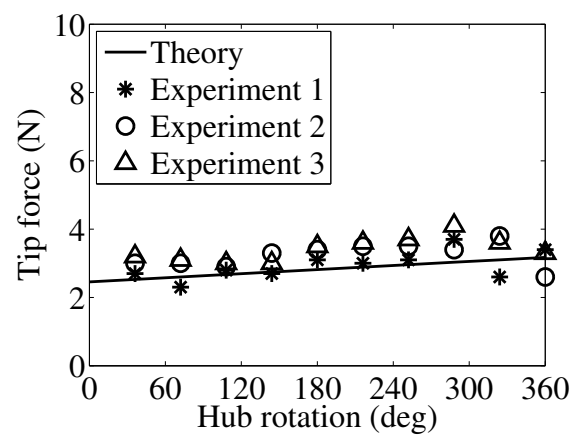

(d) $F_{\text {pre }}=4 \mathrm{~N}$

Figure 8: Tip force comparison of a 10 turns coil during blossoming $\left(\left[ \pm 47^{\circ} \mathrm{F} / 0^{\circ} / \pm 47^{\circ} \mathrm{F}\right]\right.$ (averaged), $R=20 \mathrm{~mm}, k=20 \mathrm{~N}, r_{h}=20 \mathrm{~mm}$ ) 


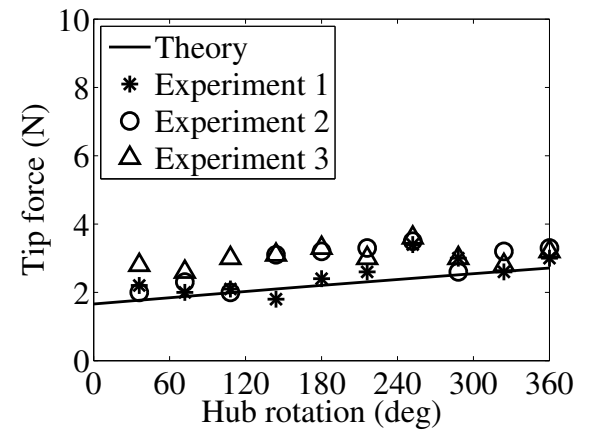

(a) $F_{\text {pre }}=1 \mathrm{~N}$

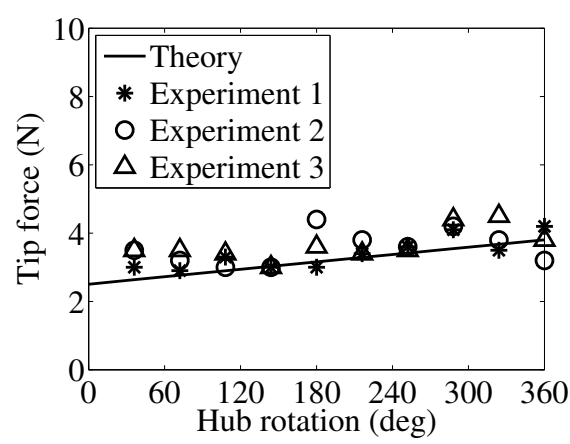

(c) $F_{\text {pre }}=3 \mathrm{~N}$

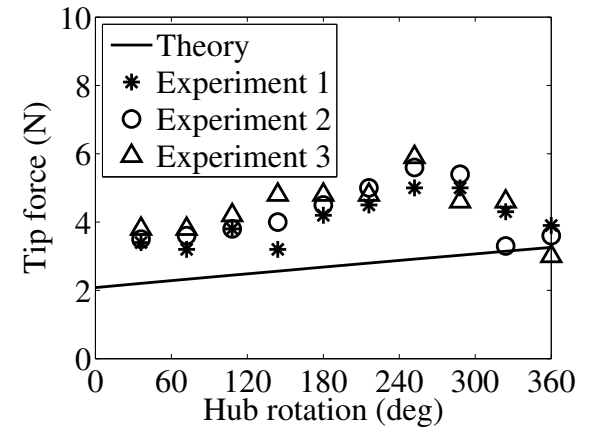

(b) $F_{\text {pre }}=2 \mathrm{~N}$

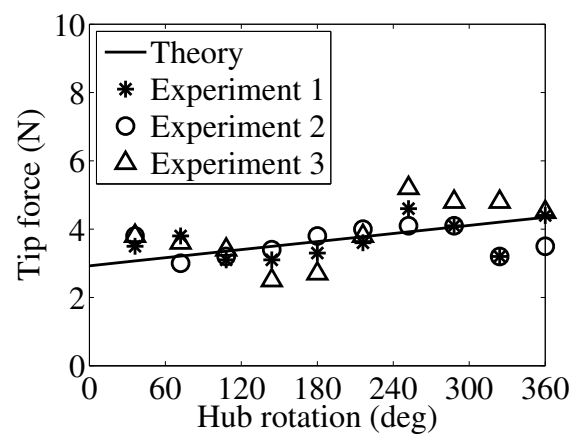

(d) $F_{\text {pre }}=4 \mathrm{~N}$

Figure 9: Tip force comparison of a 5 turns coil during blossoming $\left(\left[ \pm 48.5^{\circ} \mathrm{F} / 0^{\circ} / \pm 48.5^{\circ} \mathrm{F}\right]\right.$ (averaged), $R=20 \mathrm{~mm}, k=20 \mathrm{~N}, r_{h}=20 \mathrm{~mm}$ ) 


\section{Parametric Study}

Using the analytical method in Section 2, the influence of changing the braided angle on the boom strain energy is investigated (Figure 10), and a parametric study to explore the tip force is presented across a range of different values for the braided angle, transverse radius $R$, compression spring stiffness $k$, number of layers/turns $n_{t}$ and the hub radius $r_{h}$ values (Figure 11). Further, a tip force comparison between a bistable boom and an equivalent mono-stable boom is presented. The braided angles are held constant at $50^{\circ}$, the friction coefficient is taken as $\mu=0.1$, and remaining parameters are kept the same as those in Tables 1 and 2 (using $n_{t}=15, F_{\text {pre }}=4 \mathrm{~N}$ ). In Figure 11, any applied tip load during the deployment that is lower than those indicated in the curves would not initiate or continue blossoming.

The impact of changing the braided angle on the boom strain energy per unit length at a number of different longitudinal curvature radii values $\left(1 / \kappa_{x}\right)$ is presented in Figure 10. The strain energy can be seen to decrease approximately linearly with an increasing braided angle, so it is feasible to approximate the boom strain energy using the average angle in the calculation for the experiment in Section 3. The error introduced by taking this approach is around $1 \%$.

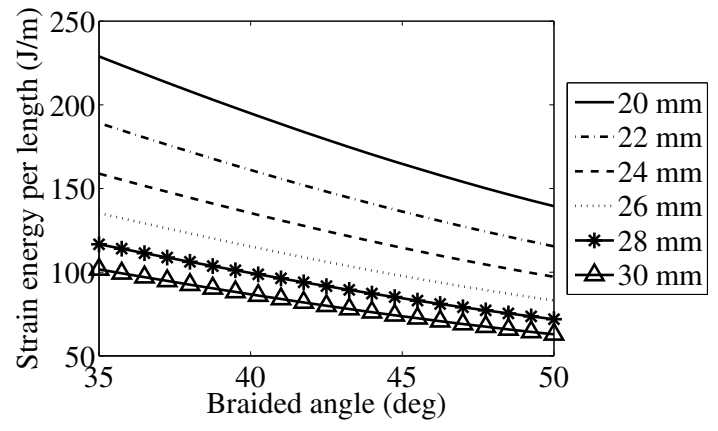

Figure 10: Strain energy as function of braided angle at different coiled radii $\left(1 / \kappa_{x}\right)$

According to Figure 11, the post-blossoming tip force increases slightly with increasing hub rotation during blossoming. From Figure 11(a), the tip force rises when the fiber angle in the braided plies increases. This is because bistable 


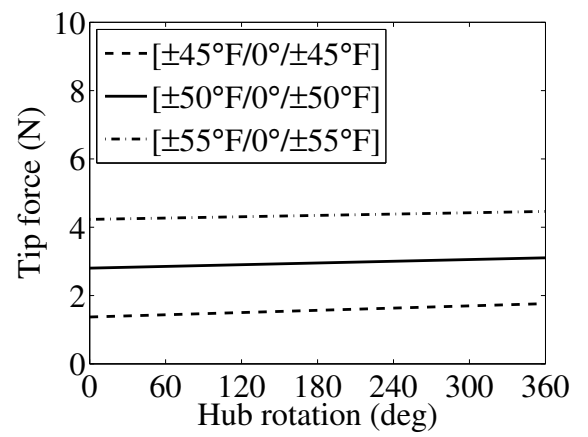

(a) varying braided angle

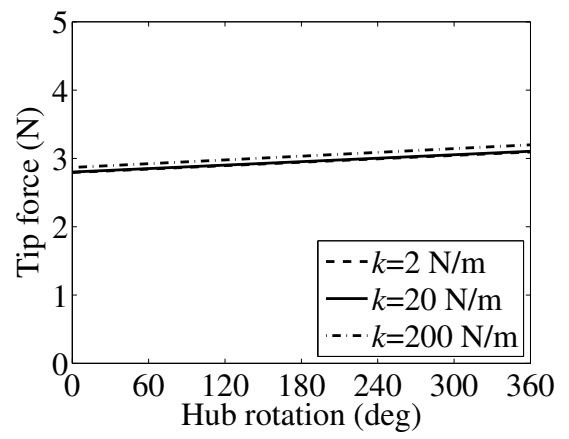

(c) varying spring stiffness

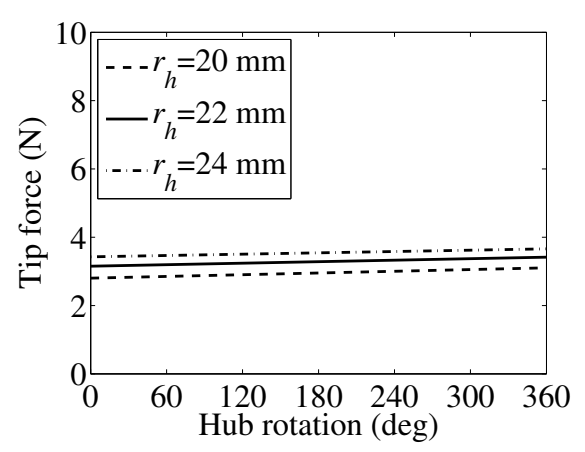

(e) varying hub radius

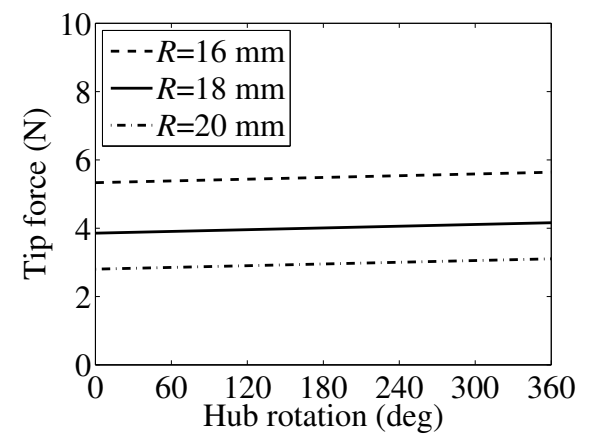

(b) varying transverse radius

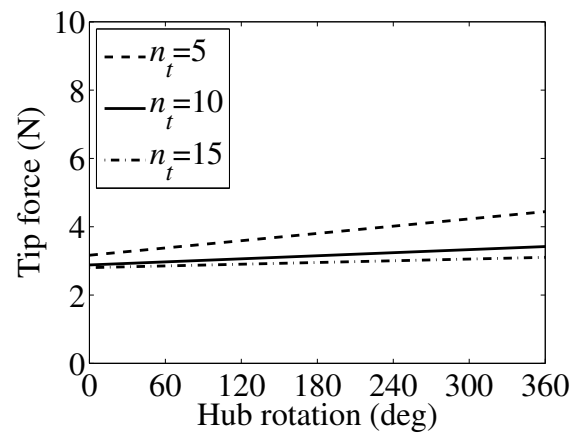

(d) varying coil layers/turns

Figure 11: Parametric analysis; unless noted, all parameters are as listed in Tables 1 and 2 (using braided angle $50^{\circ}$, number of turns $n_{t}=15$, pre-load $F_{\text {pre }}=4 \mathrm{~N}$, and friction coefficient $\mu=0.1)$. 
booms with higher fiber angles tend to have tighter natural coiled radii, with a corresponding higher energy gradient as the coiled radius increases during blossoming. In terms of continuously varying braided angle booms, the zones with higher fiber angles (the inner coil layers) can withstand higher tip loads than those with lower fiber angles (the outer coil layers), as seen in the experiments in Section 3. The same mechanism appears when the initial transverse radius $R$ is changed in Figure 11(b), as more energy is also required during coiling when the natural coiled radius is smaller. From Figures 11(a) and 11(b), both fiber angle and transverse radius have a significant influence on the tip force. Figure 11(c) confirms that the changing of compression spring stiffness $k$ has minimal effect on the tip force. The reason is that the spring stiffness terms in the analytical model (in Equations 18 and 19) are multiplied by the radial displacement of the outermost layer $\Delta r\left(\theta_{h}\right)$ which is usually small, especially for larger numbers of coil turns - see Figure 6(a).

According to the results in Figure 11(d), it can be seen that the postblossoming tip force reduces with an increasing number of turns $n_{t}$. However, the tip force (at the onset of blossoming) for different numbers of coil layers are quite similar, and hence the allowable load may be assumed constant (equal to the initial deployment state) when the number of coil layers decreases during the normal deployment process of a constant braided angle boom. For the varying braided angle booms, there is an additional effect where the average fiber angle in the coiled section increases during deployment, effectively increasing resistance to blossoming (Figures 7 to 9). Combining the analysis, for booms with a varying or constant braided angle, it can be assumed that the allowable load is the boom's tip force at its initial deployment configuration. From Figure 11(e), the hub radius $r_{h}$ does not have a great impact on the tip force results. On the basis of this, the hub radius design should be as small as possible to minimize the stowed volume needed, while also being larger than the boom natural coiled radius to reduce the blossoming torque [9].

Figure 12 shows the tip force comparison between the bistable FRP boom and a mono-stable boom (made from isotropic material) with the same longi- 
tudinal stretching stiffness $\left(A_{11}\right)$ to compare booms with the same global Euler buckling load. For the ABD matrix of the isotropic boom, the following expressions [18] are used:

$$
\begin{gathered}
A_{11}=\frac{E t}{\left(1-\nu^{2}\right)}, \\
D_{11}=D_{22}=\frac{E t^{3}}{12\left(1-\nu^{2}\right)}=D, \\
D_{12}=\nu D,
\end{gathered}
$$

The equivalent isotropic properties are $E=37.2 \mathrm{GPa}$ and $\nu=0.3$.

In Figure 12, the values above zero mean the torque/force would rotate the hub counterclockwise (i.e. acting to prevent blossoming), while those below zero mean the torque would cause the boom to blossom (rotating clockwise). Figure 12(a) shows that the tip force for the bistable boom is larger than that of the mono-stable boom. This suggests that the bistable booms are more robust in resisting blossoming than an otherwise comparable mono-stable boom. Comparing Figures 12(b) and 12(c), it can be observed that components $T_{s}$ (due to compression springs) and $T_{f}$ (due to friction) remain nominally constant, as these depend on boom geometry. For the bistable boom, as the hub radius approaches the boom natural coiled radius, $T_{b}$ approaches zero as the boom assumes its second stable state; see Figure 12(b). Based on the analysis in Section $2, T_{b}$ will be above zero if the boom coiled radius is larger than its natural coiled radius, and vice versa. In Figure 12(c), the mono-stable boom has a large negative $T_{b}$, which means the coil has a tendency to expand and blossom to assume a lower-energy configuration. This counteracts the contribution of $T_{\text {coil }}$, resulting in an overall lower resistance to blossoming compared with the bistable boom. 


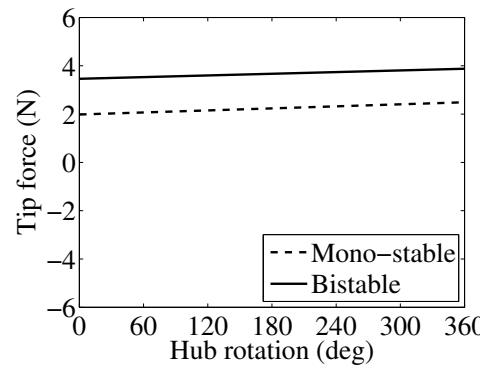

(a) tip force comparison

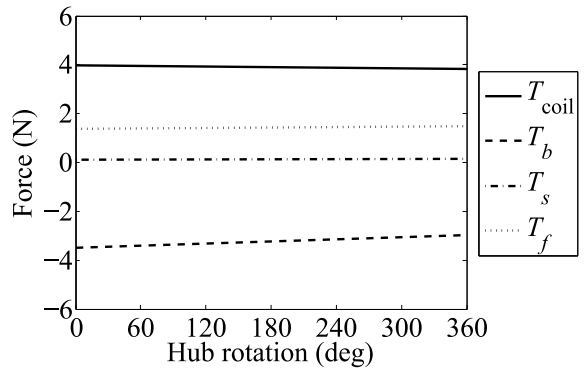

(c) mono-stable boom components

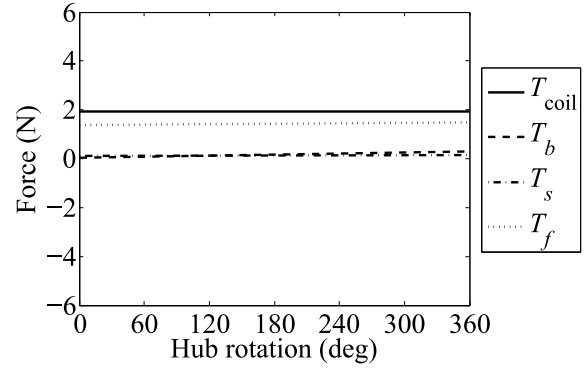

(b) bistable boom components

Figure 12: (a) Tip force for equivalent mono-stable and bistable booms; (b-c) contributions to the tip force for bistable and mono-stable booms due to the torques derived in Section 2. 


\section{Conclusion}

To be able to design a boom deployment system that works reliably, it is necessary to predict the tip force that a boom can withstand before blossoming occurs. This paper establishes a strain energy-based analytical model to capture the deployment behavior of a composite boom with a symmetric or antisymmetric layup. The shape that the coil forms around the hub was regarded as an Archimedes' spiral, and stretching energy was included into the energy balance. It was found that the analytical results were generally in good agreement with those in the experiments.

A parametric study explored the effect of the boom properties and the deployment mechanism parameters on the resistance to blossoming. An increased roller force was found to slightly increase the tip force. However, from practical experience, a high roller force can also cause local compression of the coil, and the resulting 'rolling friction' can initiate blossoming. Further, the tip force rose significantly with increasing fiber angle, and with decreasing transverse radius. The spring stiffness of the compression rollers had negligible effect on the tip force as the variation of the outermost coil layer radius was small. Further, the hub radius did not have a great impact on the tip force either; to minimise stowed volume, the hub radius should be as small as possible but larger than the natural coiled radius at the boom root. Lastly, it was concluded that the allowable load throughout the boom deployment is determined by the allowable tip force in its initial deployment configuration.

Finally, the bistable boom modelled has an improved ability to resist blossoming compared with an isotropic mono-stable boom with the equivalent Euler buckling load. This result further supports the decision to utilize bistable FRP booms for this application.

The results in this paper will help determine the tip force that an FRP composite boom can withstand in the deployment process, and provide some guidance in the design of deployable booms and controlling mechanisms. 


\section{Acknowledgements}

The authors would like to acknowledge that the research presented in this paper was carried out with the aid of National Natural Science Foundation of China (Grant No. 51835002).

\section{References}

[1] Richard I Bourgeois-Doyle. George J. Klein: The Great Inventor: NRC Press Biography Series, No. 2. NRC Research Press, 2004.

[2] CR Calladine. The theory of thin shell structures 1888-1988. Proceedings of the Institution of Mechanical Engineers, Part A: Power and Process Engineering, 202(3):141-149, 1988.

[3] Wujun Chen, Guangqiang Fang, and Yu Hu. An experimental and numerical study of flattening and wrapping process of deployable composite thin-walled lenticular tubes. Thin-Walled Structures, 111:38-47, 2017.

[4] Isaac M Daniel, Ori Ishai, Issac M Daniel, and Ishai Daniel. Engineering mechanics of composite materials, volume 3. Oxford university press New York, 1994.

[5] Andrew Daton-Lovett. Extendible member. US Patent US6217975B1, 2001.

[6] J. Fernandez, A. Viquerat, V. Lappas, and A. Daton-Lovett. Bistable over the whole length (BOWL) CFRP booms for solar sails. In Advances in Solar Sailing, pages 609-628. Springer, 2014.

[7] Juan M Fernandez. Low-cost gossamer systems for solar sailing and spacecraft deorbiting applications. PhD thesis, University of Surrey, 2015.

[8] SD Guest and S Pellegrino. Analytical models for bistable cylindrical shells. Proceedings of the Royal Society A: Mathematical, Physical and Engineering Sciences, 462(2067):839-854, 2006. 
[9] A. Hoskin. Blossoming of coiled deployable booms. PhD thesis, University of Surrey, 2018.

[10] A. Hoskin and A. Viquerat. An analysis of a coiled tape spring during compression and extension. In 3rd AIAA Spacecraft Structures Conference, San Diego, California, 4-8 Jan 2016.

[11] A. Hoskin, A. Viquerat, and G. Aglietti. Tip force blossoming of coiled deployable booms. Int. J. of Solids and Structures, 118-119:58-69, 2017.

[12] Michael W Hyer and Scott R White. Stress analysis of fiber-reinforced composite materials. DEStech Publications, Inc, 2009.

[13] K Iqbal, S Pellegrino, and A Daton-Lovett. Bi-stable composite slit tubes. In IUTAM-IASS symposium on deployable structures: theory and applications, pages 153-162. Springer, 2000.

[14] Huina Mao and Gunnar Tibert. Experiments and analytical modeling for designing tape spring composites. In 21st International Conference on Composite Materials, 2017.

[15] R. Ridenoure, R. Munakata, A. Diaz, S. Wong, Plante B., D. Stetson, D. Spencer, and J. Foley. LightSail program status: one down, one to go. In 29th Annual AIAA/USU Conference on Small Stallites, 2015.

[16] Keith Alexander Seffen, Bing Wang, and Simon David Guest. Folded orthotropic tape-springs. Journal of the Mechanics and Physics of Solids, 123:138-148, 2019.

[17] O. Stohlman and E.R. Loper. Thermal deformation of very slender triangular rollable and collapsible booms. In AIAA SciTech Conference, 2016.

[18] Stephen P Timoshenko and Sergius Woinowsky-Krieger. Theory of plates and shells. McGraw-hill, 1959.

[19] Craig Underwood, Andrew Viquerat, Mark Schenk, Ben Taylor, Chiara Massimiani, Richard Duke, Brian Stewart, Simon Fellowes, Chris Bridges, 
Guglielmo Aglietti, Berry Sanders, Davide Masutti, and Amandine Denis. Inflatesail de-orbit flight demonstration results and follow-on drag-sail applications. Acta Astronautica, 162:344-358, 2019.

[20] A. Viquerat, M. Schenk, V. Lappas, and B. Sanders. Functional and qualification testing of the InflateSail technology demonstrator. In 2nd AIAA Spacecraft Structures Conference, 5-9 January 2015, Kissimmee, FL, 2015.

[21] Sicong Wang, Mark Schenk, Hongwei Guo, and Andrew Viquerat. Tip force and pressure distribution analysis of a deployable boom during blossoming. International Journal of Solids and Structures, 192-193:141-151, 2020.

[22] Zheng Zhang, Yang Li, Xiaochen Yu, Xianghao Li, Helong Wu, Huaping Wu, Shaofei Jiang, and Guozhong Chai. Bistable morphing composite structures: a review. Thin-walled structures, 142:74-97, 2019. 